\title{
Classification and quantitative optical analysis of liquid and solid samples using a mobile phone as illumination source and detector
}

\author{
Zafar Iqbal and Mats Eriksson
}

\section{Linköping University Post Print}

\section{Tweet}

N.B.: When citing this work, cite the original article.

Original Publication:

Zafar Iqbal and Mats Eriksson, Classification and quantitative optical analysis of liquid and solid samples using a mobile phone as illumination source and detector, 2013, Sensors and actuators. B, Chemical, (185), 354-362.

http://dx.doi.org/10.1016/j.snb.2013.05.009

Copyright: Elsevier

http://www.elsevier.com/

Postprint available at: Linköping University Electronic Press

http://urn.kb.se/resolve?urn=urn:nbn:se:liu:diva-81003 


\title{
Classification and quantitative optical analysis of liquid and solid samples using a mobile phone as illumination source and detector
}

\author{
Zafar Iqbal, Mats Eriksson \\ Department of Physics, Chemistry and Biology, Linköping University, SE-581 83 Linköping, Sweden
}

\begin{abstract}
A mobile phone has been used both as illumination source and image detector for quantitative optical analysis of colored liquid samples (4 different colorants) and solid samples (printed color patterns, plastic beads and colored sand grains). Even though the measurement conditions were far from ideal, because the light source was strongly polychromatic and the illumination was not a collimated light beam, a logarithmic concentration dependence, in accordance with the Beer-Lambert law, described the data of the colored liquids quite well. By utilizing blue-blue $(420-510 \mathrm{~nm})$, green-green $(480-590 \mathrm{~nm})$ and red-red $(575-695 \mathrm{~nm})$ illumination/detection combinations, each sample could be assigned a unique color signature for classification that agreed with reference absorbance spectra measured with a spectrometer. Quantification of validation samples within a few percent of the actual values was achieved. Also the long-term repeatability of the measurements was investigated and was surprisingly good for such a simple system. Analysis of the colored solid samples was more complex with results being dependent on the morphology and colorimetric properties of the samples.
\end{abstract}

Keywords: Mobile phone; optical sensor; Beer-Lambert law; absorption; colorants

\section{Introduction}

Optical sensing by portable devices has benefited greatly in the past decade by advances in technology for instrument development [1]. Light-emitting diodes capable of providing illumination from the ultraviolet to near infrared have become the most common light source for portable instrumentation. Silicon photodiodes cover a broad spectral range as detectors and come in a variety of active area sizes and packages. Together with low-power, low-cost microprocessors the hardware available has made possible a wide range of both direct and reagent mediated analytical systems.

Analytical procedures based on consumer electronics make the technology potentially available to the general public in the area of home testing. While inexpensive electronic components in consumer products do not approach the quality necessary for use in commercial analytical instrumentation, they have demonstrated usability for optical measurements despite their limitations [2,3]. Reports of the use of digital cameras for chemical analyses, for example, have recently covered such diverse areas as detection of biogenic amines [4], pH [5], water hardness [6,7], nickel in meteorites [8] and alloy composition [9].

While consumer electronic based analytical methods are often used to only classify samples or estimate quality parameters there has also been work reported on quantitative analyses. The strategy employed in this approach was to relate the desired analytical parameter to one of the red, green or blue (RGB) color channels (or in some cases employment of a greyscale 
conversion) in order to obtain quantitative information. Reagent mediated analyses have been extensively studied in which digital images describing color development of one-shot sensing spots were evaluated $[4,6,8,10]$. There has also been work done using digital images of solutions based on their spectral properties [11-16].

Mobile phones are an attractive consumer electronic product for home testing use because of their wide availability and high level of familiarity with the general public. The digital camera associated with the phone has been the primary basis for analyses reported to date [10,17-19]. An additional feature having utility for analytical applications is the phone's screen to provide versatile sample illumination. This feature coupled with the front view camera as detector has been demonstrated to facilitate a simple spectroscopic reflectometer for qualitative analyses [20] which can be improved by image processing methods applied by the mobile phone, such as high dynamic range imaging [21].

The ability of mobile phones to achieve acceptable quantitative optical analyses is not a certainty. The 8-bit dynamic range of each RGB channel in low-cost cameras limits the detection limit [3]. There are also significant sensitivity variations among cell phone camera modules [22]. Development work in mobile liquid crystal displays (LCD) has historically been directed toward making advances in power consumption and image quality [23] and these parameters do not necessarily enhance performance as illumination source for optical analyses. Deviation from the Beer-Lambert law was in the past a commonly encountered problem when using analytical instruments exhibiting poor monochromaticity. The problem was most severe when transmittance curves of the sample and monochromator were not inversely symmetrical [24]. Modern instruments with slit widths down to $1 \mathrm{~nm}$ have remedied the situation. The measurement conditions when using the mobile phone, on the other hand, are far from those of analytical instruments. The screen's polychromaticity and the large bandwidth of the camera detector's RGB channels are considerable drawbacks. There is thus a necessity to investigate the potential of a simple "mobile phone spectrophotometer" with respect to classification, accuracy, repeatability and stability. In the study reported here the ability of a mobile phone to make quantitative determinations of colored components of liquids and solids is evaluated during periodic measurements over several days.

\section{Experimental details}

Colored liquid samples were prepared by dissolving $10 \mathrm{mg}$ of reactive blue 2 (Sigma-Aldrich, St. Louis, MO, USA), Congo red or metanil yellow (both from VWR, West Chester, PA, USA) in 1 liter de-ionized water. Dilutions were made by adding water to obtain a series of dye concentrations down to $2 \mathrm{mg} / \mathrm{l}$. Black ink was extracted from a fiber pen (Sense AB, Haninge, Sweden) and a $0.1 \mathrm{ml}$ quantity was dissolved in 1 liter de-ionized water to make a relative scale 10 solution. This was further diluted in water down to relative scale 2 black solutions. For reactive blue 2, Congo red and metanil yellow the relative concentration 10.0 corresponds to $10.0 \pm 0.2 \mathrm{mg} / \mathrm{l}$. The maximum deviations of the relative concentrations are 0.14 for all colorants for relative scales $2.00,4.00,6.00$ and 8.00 (due to the inaccuracy of the dilutions), while it is zero for relative scales 0.00 and 10.00 . The samples were prepared based on weight of colorants with no special regard for molar absorptivities. Absorbance spectra of the colored solutions were recorded using a Shimadzu (Kyoto, Japan) UV-1601PC spectrometer. 
Three different types of solid samples were studied. Colored sand samples were prepared by dissolving $200 \mathrm{mg}$ reactive blue 2 or Congo red in $100 \mathrm{ml}$ of de-ionized water. These solutions were each added to $400 \mathrm{ml}$ sand which was dried overnight at room temperature and then for 2 hours at $80^{\circ} \mathrm{C}$. A relative scale red sample series $(100=100 \%$ red sand $)$ was prepared by volumetric dilution with blue sand down to relative scale 20 . Color patterns printed on paper were designed with alternating blue and red lines (see Fig. 1c). The size of the patterned images was $4.5 \mathrm{~cm}$ times $3.7 \mathrm{~cm}$ and the line width was $0.3 \mathrm{~mm}$. Finally, red (product number 207-05) and blue (product number 207-08) plastic beads from Hama were used. The beads were in the shape of hollow cylinders, $5 \mathrm{~mm}$ high, $4.7 \mathrm{~mm}$ in diameter and with a hole with the diameter $2.7 \mathrm{~mm}$.

All samples were measured at five different occasions during a time span of 4-12 days depending on sample. Only one sample of each concentration/mixture was prepared.

Fig. 1a illustrates the measurement set-up. The photograph shows the mobile phone above the measurement vessel. The measurement vessel for the samples was a weighing boat of white polystyrene having rounded corners and dimensions 80x80x25 mm (VWR). A black laminated paper was positioned under the vessel and the phone was placed on a mechanical stand which allowed repeatable positioning and a constant distance between the sample surface and the screen/camera plane. The camera had a fixed focal length so the distance determines the field of view of the sample and in the current work $70 \mathrm{~mm}$ was a reasonable compromise for the different samples. A black, opaque plastic box was placed over the setup to exclude ambient light during measurements. All measurements were done on $70 \mathrm{ml}$ of sample.

A Nokia 6220 classic mobile phone was used as both light source and detector in the study. The phone's screen served as a controlled illumination source and the front view camera acquired information in the form of images. The secondary front view camera is designed for video calls in automatic exposure mode and has QCIF $176 \times 144$ pixels for video recording. Software was developed for phone operation. NetBean's open source Integrated Design Environment was used to design, test and implement software in the form of a MIDLet (Mobile Information Device applet) which controlled the screen as light source and simultaneously acquired images to obtain reflectance intensities from the samples.

Illuminations were performed with white light, the three primary colors red, green and blue and the three secondary colors cyan, magenta and yellow. The spectral radiances of the colors generated by the screen were measured with a standard 12 bit resolution Ocean Optics USB 2000 (Dunedin, FL, USA) fiber optics spectrophotometer. Wavelength dependent optical characteristics of the RGB channels of the phone's front view camera were measured with the Shimadzu spectrometer.

Fixed length videos were recorded by the camera at 15 frames/s with each illumination color taking $6.8 \mathrm{~s}$ of display time. Since the video capturing rate was faster than the screen illumination color rate, several frames were captured and saved for each illumination color. Consecutive series of 50 frames were selected and processed for each illumination color. Data were stored as .3gp files using H.263 video compression technology. AVS video converter6 was used to convert the .3gp mobile phone videos into image format such that each frame of the parent video file would have the same size in terms of pixels after conversion into Bitmap (bmp) image format. 
The bmp image files were loaded into MATLAB-R2007b (MathWorks, Natick, Massachusetts, USA) for image processing on a computer. The processing consisted of image data import (imread), concatenation of the 50 frames/color (strcat) and computation of RGB intensity mean values of the selected area of the image.

When liquid samples were placed in the measurement vessel and positioned under the phone, the camera recorded both specular and non-specular reflection. This is shown in Fig. $1 \mathrm{~b}$ for the liquid reference sample of de-ionized water. The solid white square at the bottom of the image was influenced by specular reflection from the sample surface as illustrated in Fig. 1a. The faint outline seen at the top and to the right of the image was the sloping bottom edge of the measurement vessel. The 60x100 pixel area in the camera's field of vision chosen to represent the samples' optical properties is shown in Fig. 1b (white, dashed rectangle). For transparent liquid samples the light detected from this direction was dominated by light that was diffusely reflected at the bottom of the vessel. This light has thus been transmitted through the liquid and was therefore strongly influenced by absorption. The mean value of the 6000 pixels of the 50 selected frames, i.e. 300000 intensity values in total, was used in the analyses. In the case of the plastic beads the influence of the specular reflection was not concentrated to a well defined area as in Fig. 1b, so the whole image (176x 144 pixels) was used to calculate the mean values in that case.

The measurement set-up shown in Fig. 1 was similar to what has been described for other portable reflectometers [25-27]. The common features were a white colored bottom for the sample cell with the surrounding surfaces being black. Light was both backscattered from the sample and bottom reflected to the detector from liquids in such a set-up. For quantitative analyses under these conditions, the relative reflectance of the diffuse reflected light recorded by the camera can be expressed as $R=I(c) / I(c=0)$, where $I(c=0)$ is the recorded intensity for pure de-ionized water. Thus $R=1$ for pure de-ionized water, just as $T=1$ for a non-absorbing liquid in a transmission experiment. The optical density of the sample is $A=-\ln R$ by analogy to the relationship between transmittance and absorbance [25].

A number of calibration plots can be considered for relating reflection in the non-specular part of the image to sample concentration, $c$ [27]. A simple linear relationship between $R$ and $c$ can be suitable for samples exhibiting low absorbance or where scattering is the dominant mechanism. A logarithmic relationship, in accordance with the Beer-Lambert law should become important when considerable light attenuation by sample absorbance is encountered.

\section{Results and discussion}

A first consideration in evaluating the mobile phone's performance for quantitative optical analyses is the relationship between the screen illumination and the output RGB signals from each camera pixel. Fig. 2 a shows the intensities of RGB colors from the screen as recorded by the Ocean-Optics spectrometer. Green was the dominant color emitted and a wide spectral range was observed for both red, green and blue illumination, as expected. The CMOS photodetector of camera systems consists of pixels covered by a red, green or blue filter in a specific pattern, usually the Bayer color filter array. The output RGB signals for each pixel undergo color interpolation and correction by the camera of the raw photoelectron current generated at each pixel. The camera's response as a function of wavelength was determined during illumination from the Shimadzu spectrometer is shown in Fig. 2b. The bandwidth for each channel was fairly wide with considerable overlap between colors. Multiplying these 
wavelength dependent responses of the camera by the corresponding screen illumination intensities yielded the effective channel response spectra shown in Fig. 2c. The letters beside the spectra indicate which illumination/detection channel combination that is considered. The capital letter indicates the illumination color $(\mathrm{R}=\mathrm{red}, \mathrm{G}=$ green or $\mathrm{B}=$ blue $)$ and the lower case letter the detection channel ( $\mathrm{r}, \mathrm{g}$ or $\mathrm{b})$. The spectra illustrate the expected resolution in the RGB camera channels when using the phone's screen as illumination source and the frontview camera as detector. Arbitrarily choosing the 0.1 level for defining the spectral range of each channel, indicates that sensitivity for blue was at 420-510 nm, green at 480-590 and red at $575-695 \mathrm{~nm}$.

Fig. 2 illustrates the limitations of using the mobile phone as an analytical device for quantitative analysis based on the Beer-Lambert law:

$$
T=I / I_{0}=10^{-\varepsilon l c}
$$

where $T$ is the transmission of light with incident intensity $I_{0}$ and transmitted intensity $I, \varepsilon$ is the molar absorptivity, $l$ is the path length through the liquid and $c$ is the concentration. The law is valid only when identical photons (monochromatic radiation) interact with the sample under study. The condition is impossible to meet in practice even using single line sources of illumination. Common laboratory spectrometers using grating monochromators can have polychromatic radiation leading to assay errors of up to $4 \%$ even for absorption levels $<1$ [28]. The severity of the problem is also related to the variation of the molar absorptivity of the sample as a function of wavelength in the region where the measurement is made [29]. As seen in Fig. 2c the wavelength region can be fairly broad for the mobile phone analysis. One way to narrow the wavelength region with the mobile phone is to combine illumination and image sensor channels of different color. This is illustrated in Fig. $2 \mathrm{~d}$ for the cases of green illumination/blue image sensor channel $(\mathrm{Gb})$ and green illumination/red image sensor channel (Gr), respectively. Note that these combinations also give rise to a maximum effective response at wavelengths that differ from those of $\mathrm{Rr}, \mathrm{Gg}$ and $\mathrm{Bb}$.

\subsection{Colored solutions}

The liquid samples chosen for investigation covered a range of colors. Reference absorbance spectra measured with a spectrometer are shown in Fig. 3. Reactive blue 2 had an absorbance maximum at about $610 \mathrm{~nm}$, Congo red at about $500 \mathrm{~nm}$ and metanil yellow at about $430 \mathrm{~nm}$. Black ink exhibited three absorbance maxima at about 630, 510 and $410 \mathrm{~nm}$ corresponding to its black color property of absorbing light over the entire visible spectrum. The peak widths at half height for the maxima were thus greater than the $20-50 \mathrm{~nm}$ range required for maintaining a constant molar absorptivity using filters found in colorimeters [29]. The mobile phone typically exhibited less favorable conditions as shown in Fig. 2c, even if this can be remedied to some extent as shown in Fig. 2d, though at the expense of much lower effective response (not shown in the figure).

Figs. 4 a-c show data measured with the mobile phone, that are based on reflected intensities for three colored solutions using recorded intensities from the combinations red illumination/red channel $(\mathrm{Rr})$, green illumination/green channel $(\mathrm{Gg})$ and blue illumination/blue channel $(\mathrm{Bb})$. The relative reflectance is the measured intensity, $I$, divided by the mean intensity for de-ionized water, $I_{0}$. The strongest decrease of detected light 
intensity with increasing concentration followed the absorbance spectra shown in Fig. 3, red for reactive blue 2, green/blue for Congo red and blue for metanil yellow. Furthermore, there was also a good correlation between all observed $\mathrm{Rr}, \mathrm{Gg}$ and $\mathrm{Bb}$ values measured by the mobile phone and the reference absorbance spectra. It should be noted that metanil yellow has a much lower molecular weight and higher molar absorptivity than the other two dyes which resulted in the lower reflection intensities from its solutions for the same weight designated concentrations.

In general the data was strongly non-linear in cases where a strong absorption occurred. This is seen by comparing with the linear curve fits in these cases. A linearization of the plots with logarithmic functions was attempted. Plotting $B L=\ln \left(I_{o} / I\right)$ versus concentration, in accordance with the Beer-Lambert law, eq. (1), gave a significantly improved linear concentration dependence as expected. It was also observed that, for the highest concentrations of metanil yellow in the case of blue illumination/blue channel $(\mathrm{Bb})$, the linearity could be further improved by plotting

$$
B L_{m}=\ln \left(I_{o}-I_{1}\right) /\left(I-I_{l}\right)
$$

against concentration, where $B L_{m}$ is a modified Beer-Lambert expression and $I_{l}$ is an empirically fitted constant. For the $\mathrm{Gg}$ and $\mathrm{Rr}$ conditions of this study the absorption was not as high, so the Beer-Lambert expression was used without modification in those cases (i.e. $I_{l}=0$ and $B L_{m}=B L$ for $\mathrm{Gg}$ and $\mathrm{Rr}$ ). The results for the three solutions are shown in Fig. $4 \mathrm{~d}-\mathrm{f}$.

The good linearity of the Beer-Lambert expression (slightly modified in the case of metanil yellow) is surprising since the measurement conditions were far from ideal, with a light source that was strongly polychromatic, an illumination that was not a collimated light beam with homogeneous light intensity and with different path lengths through the liquid for different pixels of the screen. The need of the constant $I_{l}$ for the highest concentrations of metanil yellow during $\mathrm{Bb}$ conditions might be due to light scattering.

The standard deviation was quite small among the 5 repetitions of each measurement, even though the measurement period lasted for up to nine days and the different concentrations were studied in randomized order. The measurement technique thus provides a good repeatability for a fairly long time.

The data shown in Fig. 4 for the different illumination/camera channel combinations provided a unique fingerprint for each solution. They were used for a 3-D quantitative visualization of each solution as a function of concentration as shown in Fig. 5. Here the three defining Rr, Gg and $\mathrm{Bb}$ values (i.e. the $B L_{m}$ values of Fig. $4 \mathrm{~d}-\mathrm{f}$ ) for each concentration were used as coordinates when constructing the figure. The figure illustrates that the data can be used both for classification and for quantification. The direction in RGB space (or $\mathrm{RrGgBb}$ space) is clearly different for the three different solutions and the distance from the origin (i.e. $\sqrt{R r^{2}+G g^{2}+B b^{2}}$ ) increases proportionally with increasing concentration.

RGB calibration curves were obtained by fitting a straight line (least squares fit passing through the origin) to the data (obtained from the distances from the origin in RGB space) for the different colorants, respectively. The results are shown in Fig. 6. The use of composite RGB curves was especially useful for the black ink solutions (Fig. 6d) since these absorbed all colors. These calibration curves were used in the validation experiments of unknown concentrations presented below. In Fig. 6 both the standard deviation of the mobile phone 
measurements (vertical error bars) and the maximum deviations due to uncertainties of the relative concentrations (horizontal error bars) are included. The calibration curves fit many of the data points within the limits of the uncertainties. There are, however, several exceptions indicating that there might be nonlinear terms contributing to the concentration dependence. We have restricted ourselves to a simple linear regression in this case though.

A detection limit for reactive blue 2, Congo red and metanil yellow of $0.2 \pm 0.1 \mathrm{mg} / \mathrm{l} \mathrm{can} \mathrm{be}$ estimated from the data, based on three times the standard deviation at zero concentration (lower value) and at relative concentration 2.0 (higher value), respectively.

It should be mentioned that similar measurements of the $r, g$ and $b$ channel intensities during white illumination were also performed. Those results were, however, not as straightforward to evaluate and we therefore omit them in this presentation.

\subsection{Solid samples}

Solid samples presented a different optical model from the liquids in that the illuminating light was absorbed, scattered and reflected from only the sample surface. Three types of solid samples were studied: paper samples with printed lines $(0.3 \mathrm{~mm}$ line width) of alternating blue and red color in different proportions, red and blue plastic beads and, finally, colored sand grains. The sand was colored by impregnation with Congo red or reactive blue 2 solutions at 200 times the concentrations used to obtain the spectra in Fig. 3. The pure red and blue samples in the series thus had absorbance maxima at about 500 and $610 \mathrm{~nm}$, respectively. While the paper samples were flat, the plastic beads introduced a much rougher surface with shadows and irregular specular reflections that hampered the evaluation of the images. The sand grains were not completely covered by colored ink and thus superimposed their natural color to the red and blue ink and therefore made the measurement conditions even harder.

Fig. 7 shows reflectance curves for the solid samples. Figs. 7a and b compare the $\mathrm{Rr}$ and $\mathrm{Bb}$ illumination/detection combinations for the paper samples with the $\mathrm{Mr}$ and $\mathrm{Mb}$ combinations, respectively, where $\mathrm{M}$ stands for magenta (red + blue) illumination. Both $\mathrm{Rr}$ and $\mathrm{Mr}$ followed the increase in red color quite well. The performance of $\mathrm{Mb}$ was, however, much better than that of $\mathrm{Bb}$ versus decreasing blue color. Therefore the rest of the samples were studied only with the magenta illumination and detection with the red and blue channels.

In the case of the plastic beads, Fig. 7c and d, the linearity was not as good as for the paper samples, but it was still possible to classify the different concentrations of the samples. For the sand grains, however, it started getting very difficult to distinguish samples with about the same concentrations of red and blue grains, i.e. 40-60 and 60-40, for Mr conditions, as shown in Fig. 7e. There was (almost) a threshold concentration for reflection of the red part of the magenta illumination at about the $60 \%$ relative concentration level. For the Mb measurement conditions almost no classification was possible, as shown in Fig. 7f. Also the repeatability of the sand grain measurements was poor as indicated by the large error bars.

In general the Mr illumination/detection combination showed the best results. In the case of the colored sand grains a non-linear calibration curve might be considered. One possibility to improve the results of the plastic beads and the sand grains might be to use different illumination angles combined with more advanced image analysis. 


\subsection{Validation measurements}

Validation measurements were performed in randomized order, with three additional samples for each sample category. For the colored liquids the calibration curves shown in Fig. 6 were used and for the solid samples the Mr calibration curves were used. Actual values, mean values, errors and standard deviation (std) values obtained from five measurements of each concentration of three liquid samples are shown in Table 1 and of the three solid samples in Table 2.

Generally good agreement was obtained between the measured and actual concentrations for the colored liquid samples. Also the colored paper samples gave good validation results, while relatively large errors were observed for some of the plastic bead and sand grain samples, which could be attributed to the complexity of these samples and is in agreement with the smaller $\mathrm{R}^{2}$ values for the calibration curves of these samples.

\section{Conclusions}

A study of the potential of using a mobile phone for simple spectrophotometric measurements has been performed. It is found that both liquid and solid samples can be classified as well as quantified based on their color information in a few simple cases.

The colored liquid samples were well described by an exponential decrease of the measured light intensity with increasing concentration and only a small adjustment of the Beer-Lambert expression was needed in order to linearize the data. This is a remarkable result considering the strongly non-ideal measurement conditions (a widely polychromatic light source, varying light path lengths through the liquid and a non-collimated light beam with non-homogeneous light intensity). By combining data from measurement combinations with red illumination/red detection channel, green illumination/green detection channel and blue illumination/blue detection channel, the different colorants were readily classified and different concentrations quantified with a precision better than a few percent in most cases. Each color of the investigated liquid solutions gave a unique fingerprint (direction) in the three dimensional RGB space. The measurements were repeated during time spans of 4-12 days depending on sample, with low variance and therefore good repeatability.

The possibility to classify and quantify the solid samples depended on the complexity of the samples. Flat paper samples with clear blue and red lines proved to be simple cases, while plastic beads with a much coarser surface and irregular shadows and areas with specular reflection gave rise to larger measurement errors. In the case of partly colored sand grains a non-linear calibration curve was obtained and a larger variance of the results hampered quantifications, in particular for equimixtures of the two colors.

In this study colored samples have been investigated. In an ongoing study also non-colored transparent liquid samples are investigated with the same technique, but with a different evaluation procedure and the results will be the subject of a separate paper.

\section{Acknowledgements}

Zafar Iqbal is recipient of a scholarship from Pakistan's Higher Education Commission (HEC). The authors thank Dr. Robert Bjorklund for his valuable contributions to the work. 


\section{References}

[1] L.F. Capitán-Vallvey, A. J. Palma, Recent developments in handheld and portable optosensing - A review, Anal. Chim. Acta 696 (2011) 27-46.

[2] N. A. Rakow, K. S. Suslick, A Colorimetric Sensor Array for Odor Visualization, Nature 406 (2000) 710-713.

[3] A. Scheeline, Teaching, learning, and using spectroscopy with commercial, off-the-shelf technology, Appl. Spec. 64 (2010) 256A-268A.

[4] M.-S. Steiner, R. J. Meier, A. Duerkop, O.S. Wolfbeis, Chromogenic sensing of biogenic amines using a chameleon probe and the red-green-blue readout of digital camera images, Anal. Chem. 82 (2010) 8402-8405.

[5] Savafi, N. Maleki, A. Rostamzadeh, S. Maesum, CCD camera full range $\mathrm{pH}$ sensor array, Talanta (2007) 498-501.

[6] A. Lapresta-Fernández, L.F.Capitán-Vallvey, Multi-ion detection by one-shot optical sensors using a colour digital photographic camera, Analyst 136 (2011) 3917-3926.

[7] Lopez-Molinero, V. J. Cubero, R. D. Irigoyen, D. S. Piazuelo, Feasibility of digital image colorimetry-Application for water calcium hardness determination, Talanta (2013) 236-244.

[8] L.L. Zamora, P.A. López, G.M.A. Fos, R.M. Algarra, A.M.M. Romero, J.M. Calatayud, Quantitative colorimetric-imaging analysis of nickel in iron meteorites, Talanta, 83 (2011) 1575-1579.

[9] N. Maleki, A. Savafi, F. Sedaghatpour, Single-step calibration, prediction and real samples data acquisition for artificial neural network using a CCD camera, Talanta (2004) 830-835.

[10] A.W. Martinez, S.T. Phillips, E. Carrilho, S.W. Thomas, H. Sindi, G.M. Whitesides, Simple telemedicine for developing regions: camera phones and paper-based microfluidic devices for real-time, off-site diagnosis, Anal. Chem. 80 (2008) 3699-3707.

[11] M. Santonico, A. Scozzari, G. Brozzo, L. Marinib, A. D’Amico, D. Filippini, I. Lundström, C. Di Natale, Detection of Natural Cr(VI) with Computer Screen Photoassisted Technology, Procedia Chem. 1 (2009) 317-320.

[12] A. R. Tôrres, W. da Silva Lyra, S.I.E. de Andrade, R. A. N. Andrade, E. C. da Silva, M.C.U. Araújo, E. da Nóbrega Gaião, A digital image-based method for determining of total acidity in red wines using acid-base titration without indicator, Talanta 84 (2011) 601-606. 
[13] S. K. Kohl, J. D. Landmark, D. F. Stickle, Demonstration of absorbance using digital color image analysis and colored solutions, Journal of Chemical Education (2006) 644646.

[14] E. N. Gaiao, V. L. Martins, W. S. Lyra, L. F. Almeida, E. C. Silva, M. C. U. Araújo, Digital image-based titrations, Analytica Chimica Acta (2006) 283-290.

[15] W. Wongwilai, S. Lapanantnoppakhun, S. Grudpan, K. Grudpan, Webcam camera as a detector for a simple lab-on-chip time based approach, Talanta 81 (2010) 1137-1141.

[16] P: M. B. Lima, S. I. E. Andrade, I. S. Barreto, L. F. Almeida, M. C. U. Araújo, A digital image-based micro-flow-batch analyzer, Microchemical Journal 106 (2013) 238-243.

[17] B.A. Cadle, K.C. Rasmus, J.A. Varela, L.S. Leverich, C.E. O’Neill, R.K. Bachtell, D.C. Cooper, Cellular phone-based image acquisition and quantitative ratiometric method for detecting cocaine and benzoylecgonine for biological and forensic applications, Subst. Abuse: Res. Treat. 4 (2010) 21-33.

[18] H.P. Ninh, Y. Tanaka, T. Nakamoto, K. Hamada, A bad-smell sensing network using gas detector tubes and mobile phone cameras, Sens. Actuators B 125 (2007) 138-143.

[19] A. García, M.M. Erenas, E. D. Marinetto, C. A. Abad, I. de Orbe-Paya, A. J. Palma, L. F. Capitán-Vallvey, Mobile phone platform as portable chemical analyzer, Sens. Actuators B 156 (2011) 350-359.

[20] Z. Iqbal, R.B. Bjorklund, Assessment of a mobile phone for use as a spectroscopic analytical tool for foods and beverages, Int. J. Food Sci. Technol. 46 (2011) 2428-2436.

[21] P. Preechaburana, S. Macken, A. Suska, D. Filippini, HDR imaging evaluation of a NTproBNP test with a mobile phone, Biosens. Bioelectron. 26 (2011) 2107-2113.

[22] R. Safaee-Rad, M. Aleksic, An efficient spectral-based calibration method for RGB white-balancing gains under various illumination conditions for cell-phone cameras, Proc. SPIE 7536, Sensors, Cameras, and Systems for Industrial/Scientific Applications XI (2010) $75360 \mathrm{U}$

[23] Y.J. Kim, M.R. Luo, W. Choe, H.S. Kim, S.O. Park, Y. Baek, P. Rhodes, S. Lee, C.Y. Kim, Factors affecting the psychophysical image quality evaluation of mobile phone displays: the case of transmissive liquid-crystal displays, J. Opt. Soc. Am. A 25(2008) 2215-2222.

[24] H. Goldenberg, Rectification of Nonlinear Beer's law Plots - Application to Alkaline Chromate and p-Nitrophenolate Solutions, Anal. Chem. 26 (1954) 690-693.

[25] F.A.A. Matias, M. M.D.C. Vila, M. Tubino, A simple device for quantitative colorimetric diffuse reflectance measurements, Sens. Actuators B 88 (2003) 60-66.

[26] K.-T. Lau, W.S. Yerazunis, R.L. Shepherd, D. Diamond, Quantitative colorimetric analysis of dye mixtures using an optical photometer based on LED array, Sens. Actuators B 114 (2006) 819-825. 
[27] M. Tubino, R.L. de Souza, Determination of diclofenac in pharmaceutical preparations by diffuse reflectance photometry, Talanta 68 (2006) 776-780.

[28] C. Galli, Modeling systematic errors: polychromatic sources of Beer-Lambert deviations in HPLC/UV and nonchromatographic spectrophotometric assays, J. Pharm. Biomed. Anal., 25 (2001) 803-809.

[29] W.E. Wentworth, Dependence of the Beer-Lambert absorption law on monochromatic radiation - An experiment in spectrophotometry, J. Chem. Educ. 43 (1966) 262-264.

\section{Biographies}

Zafar Iqbal received his MSc degree in Physics (major in experimental physics, from the University of Punjab, Lahore, Pakistan) and in Engineering (major in design engineering, from Quaid-e-Azam University, Islamabad, Pakistan) in the years 1988 and 1992, respectively. During 1992-2007 he worked as principal scientist at the department of design engineering at the Pakistan atomic energy commission (PAEC), with mathematical-modeling, software-development and computer-simulations with respect to system design and design evaluation. He has since 2007 been with Linköping University working on different aspects of optical sensing with mobile phones and received his $\mathrm{PhD}$ in 2012.

Mats Eriksson is Associate Professor at Linköping University, Sweden. Eriksson received his PhD in Applied physics in 1997. He is since 2007 heading a chemical sensor group and was during 2006-2009 working part time as a Senior Scientist at AppliedSensor. His research interest is primarily on chemical sensors applied to water monitoring and different aspects of gas sensing, in particular with field-effect devices, covering areas such as electrochemistry, surface and interface physics, catalytic reactions, thin film physics, semiconductor physics and optical sensing. He is currently involved in projects on electronic tongues and microelectrode arrays for drinking water monitoring.

\section{Figure captions}

Fig. 1. a) Experimental set-up viewed from the side (left) and obliquely from above (inset) with the mobile phone on a mechanical stand above the measurement vessel. The drawing indicates the area where specular reflection from liquid surfaces is expected. b) One of the recorded frames during measurements of clean water. The solid white area is influenced by specular reflection. The dashed white rectangle shows the area used for analysis. c) The pattern used for $40 \%$ red (black in the pattern) and $60 \%$ blue (white in the pattern) of the paper version of the solid samples. 
Fig. 2. (a) Spectral emission for red (R, solid), green ( $\mathrm{G}$, dash) and blue (B, dot ) illumination from the screen. (b) Normalized channel response for the camera for the red (r), green (g) and blue (b) channels, respectively. (c) Effective channel response spectra (screen illumination intensity times camera's channel response) for illumination/image channel combinations $\mathrm{Rr}$, $\mathrm{Gg}$ and $\mathrm{Bb}$ and (d) for illumination/image channel combinations $\mathrm{Gb}$ and $\mathrm{Gr}$.

Fig. 3. Absorbance spectra for metanil yellow (solid), Congo red (dash dot), reactive blue 2 (dot) at $10 \mathrm{mg} / \mathrm{l}$ concentrations, and black ink (dash) at relative scale 10 concentration. The path length in the liquids is $1 \mathrm{~cm}$.

Fig. 4. Relative reflectance data and linear curve fits (in the least squares sense) for light recorded in the red channel during red illumination $(\mathrm{Rr})$, green channel during green illumination $(\mathrm{Gg})$, and blue channel during blue illumination $(\mathrm{Bb})$, for $(\mathrm{a})$ reactive blue $2,(\mathrm{~b})$ Congo red and (c) metanil yellow solutions. Data points are from the average of 5 measurements taken on all samples over 8, 4 and 9 day periods, for the different colorants respectively. The standard deviation is also shown, but it is smaller than the marker size in most cases. (d-f) Linearization of the data using the function $B L_{m}=\ln \left(I_{o}-I_{1}\right) /\left(I-I_{1}\right)$ where $I_{1}$ is an empirically fitted constant accounting for deviations from the Beer-Lambert law at the highest metanil yellow concentrations for the $\mathrm{Bb}$ data $\left(I_{l}=0\right.$ for the $\mathrm{Rr}$ and $\mathrm{Gg}$ data). The $\mathrm{R}^{2}$ values are coefficients of determination, a goodness value of the curve fit.

Fig. 5. Quantitative 3-D description of the three colored solutions with the logarithmic values shown in Fig. $4 d-f$ as coordinates. Concentration radiates away from the origin $(0,0,0)$. The data of the highest concentrations are plotted so that they cross the three different planes of the bounding box not in contact with the origin (called "tight axis" in Matlab).

Fig. 6. Calibration curves based on distance from the origin in $\mathrm{RrGgBb}$ space (see Fig. 5) for illumination/camera channels $\mathrm{Rr}, \mathrm{Gg}$ and $\mathrm{Bb}$ for (a) reactive blue 2, (b) Congo red,(c) metanil yellow and (d) black ink solutions. Mean values and standard deviations (vertical error bars) are shown for five measurements conducted during 8, 4, 9 and 12 days for the four different colorants, respectively. Also maximum deviations in the relative concentrations are indicated (horizontal error bars).

Fig. 7. Relative reflectance of colored solid samples ( 0 corresponds to $100 \%$ blue color and 100 corresponds to $100 \%$ red color). Data of colored paper samples are shown for (a) $\mathrm{Rr}$ and $\mathrm{Mr}$ illumination/detection combinations and (b) for $\mathrm{Bb}$ and $\mathrm{Mb}$ combinations. Data of colored plastic beads for (c) Mr and (d) Mb measurement conditions. Data of colored sand grains for (e) $\mathrm{Mr}$ and (f) $\mathrm{Mb}$ measurement conditions. 
Table 1 . Validation measurements of the liquid samples.

\begin{tabular}{|l|c|c|c|c|c|c|c|c|c|}
\hline & \multicolumn{3}{|c|}{ Reactive blue 2 } & \multicolumn{3}{c|}{ Metanil yellow } & \multicolumn{3}{c|}{ Black ink } \\
\hline Actual value & 3.0 & 5.0 & 8.0 & 1.0 & 3.0 & 9.0 & 1.0 & 5.0 & 8.0 \\
\hline Measured value & 3.0 & 4.9 & 7.8 & 0.8 & 3.0 & 9.0 & 1.0 & 4.8 & 7.8 \\
\hline Error & 0.0 & -0.1 & -0.2 & -0.2 & 0.0 & 0.0 & 0.0 & -0.2 & -0.2 \\
\hline Standard deviation & 0.14 & 0.07 & 0.15 & 0.05 & 0.15 & 0.38 & 0.02 & 0.01 & 0.32 \\
\hline
\end{tabular}


Table 2 . Validation measurements of the solid samples.

\begin{tabular}{|l|c|c|c|c|c|c|c|c|c|}
\hline & \multicolumn{3}{|c|}{ Colored paper } & \multicolumn{3}{c|}{ Plastic beads } & \multicolumn{3}{c|}{ Colored sand grains } \\
\hline Actual value & 10 & 50 & 90 & 10 & 50 & 90 & 30 & 50 & 90 \\
\hline Measured value & 11 & 52 & 90 & 16 & 50 & 81 & 23 & 61 & 94 \\
\hline Error & 1 & 2 & 0 & 6 & 0 & -9 & -7 & 11 & 4 \\
\hline Standard deviation & 0.4 & 0.8 & 0.3 & 0.8 & 0.8 & 0.9 & 13 & 10 & 0.7 \\
\hline
\end{tabular}


a)

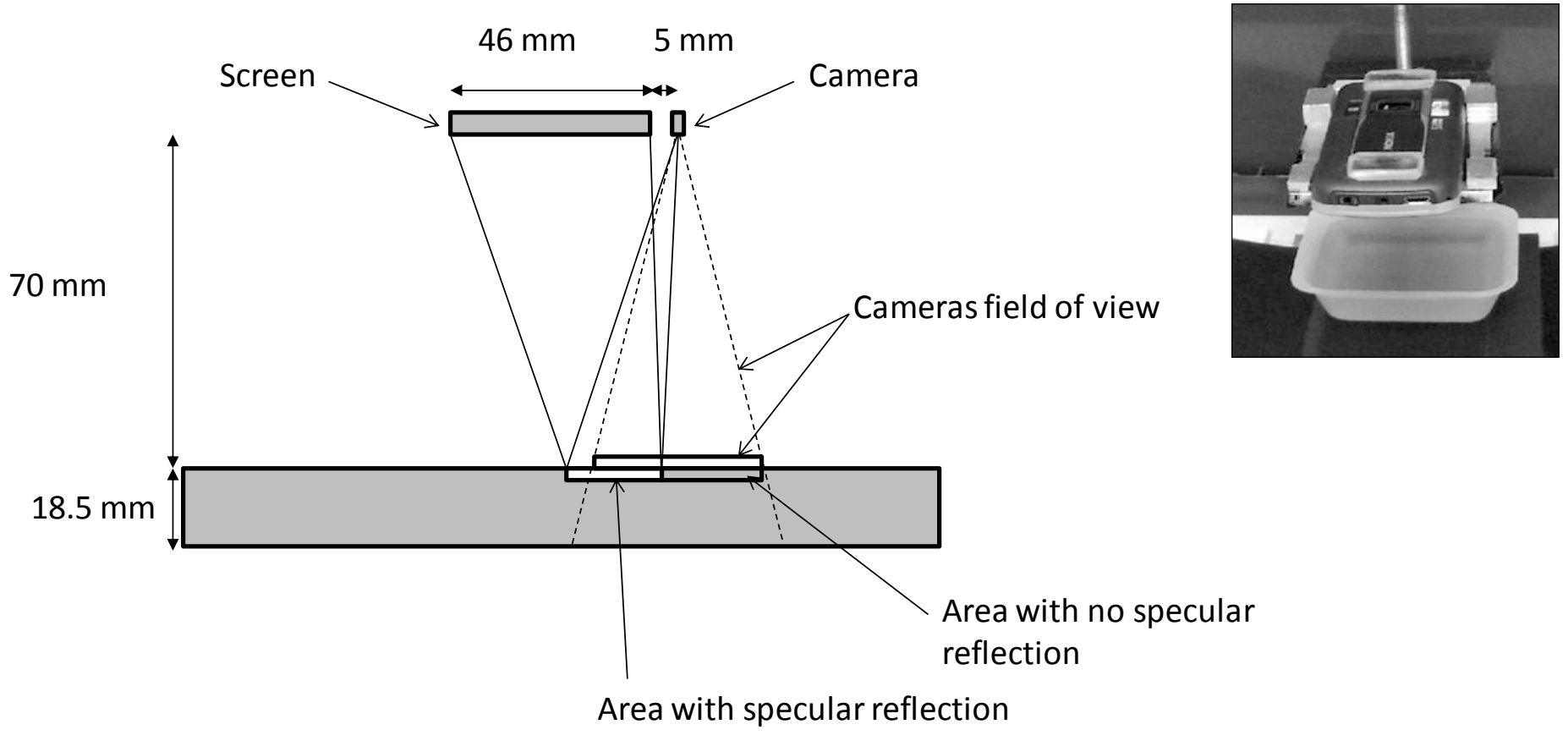

b)

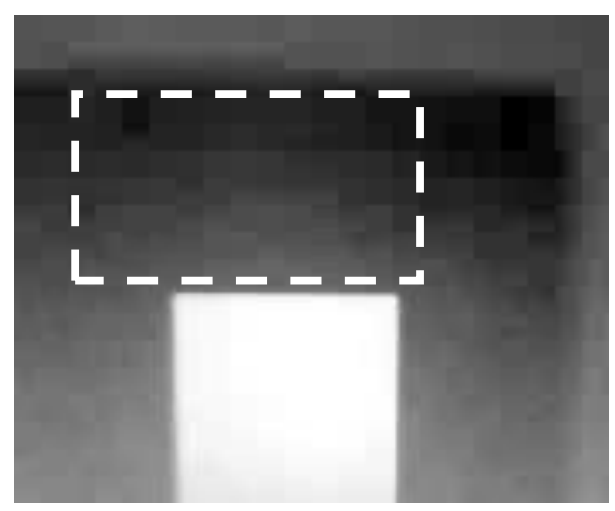

c)

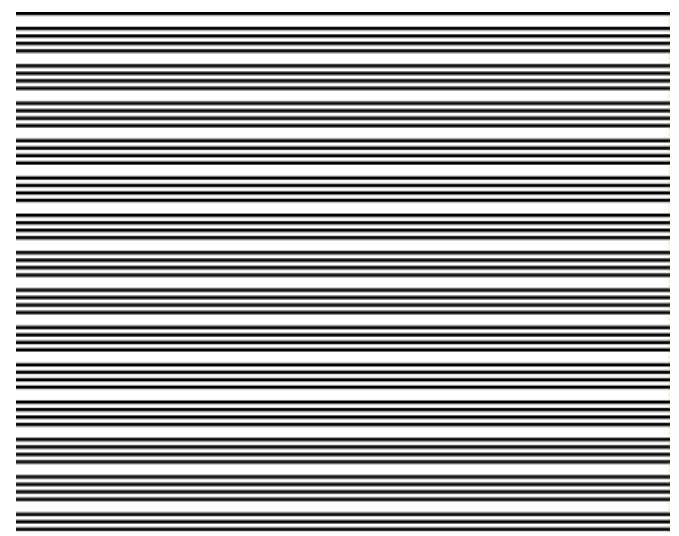


a)

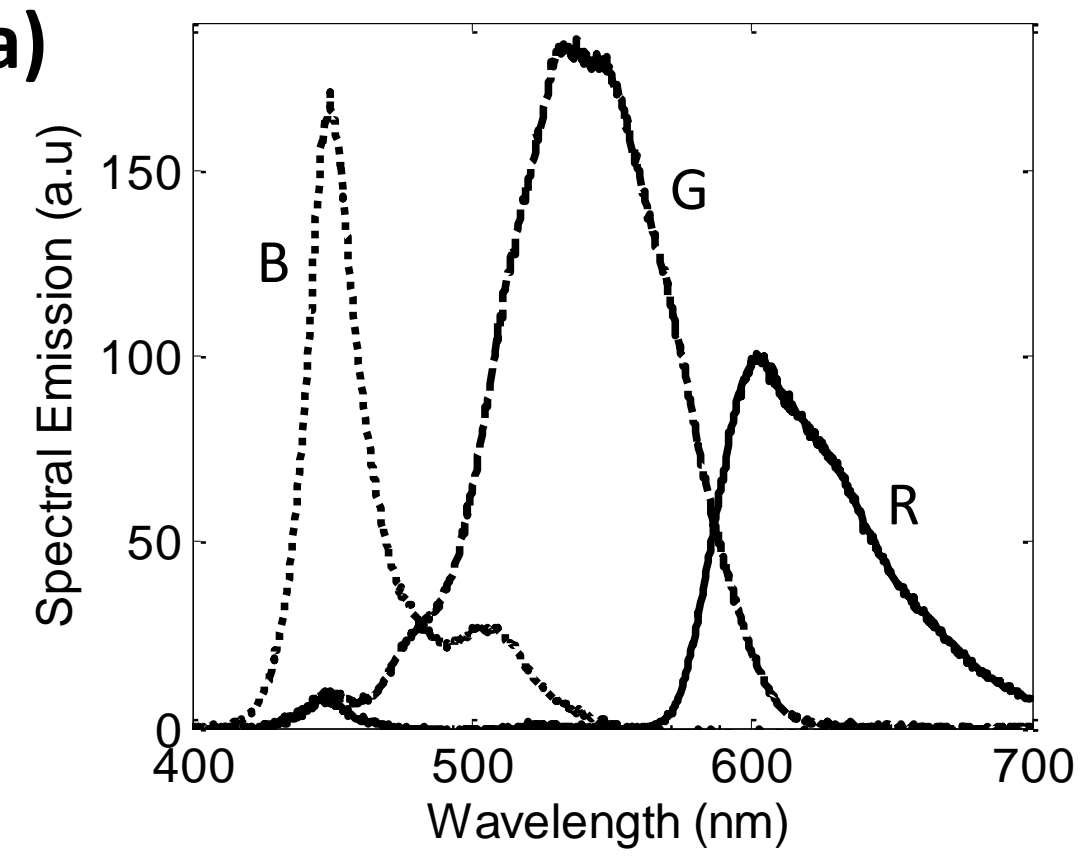

c)

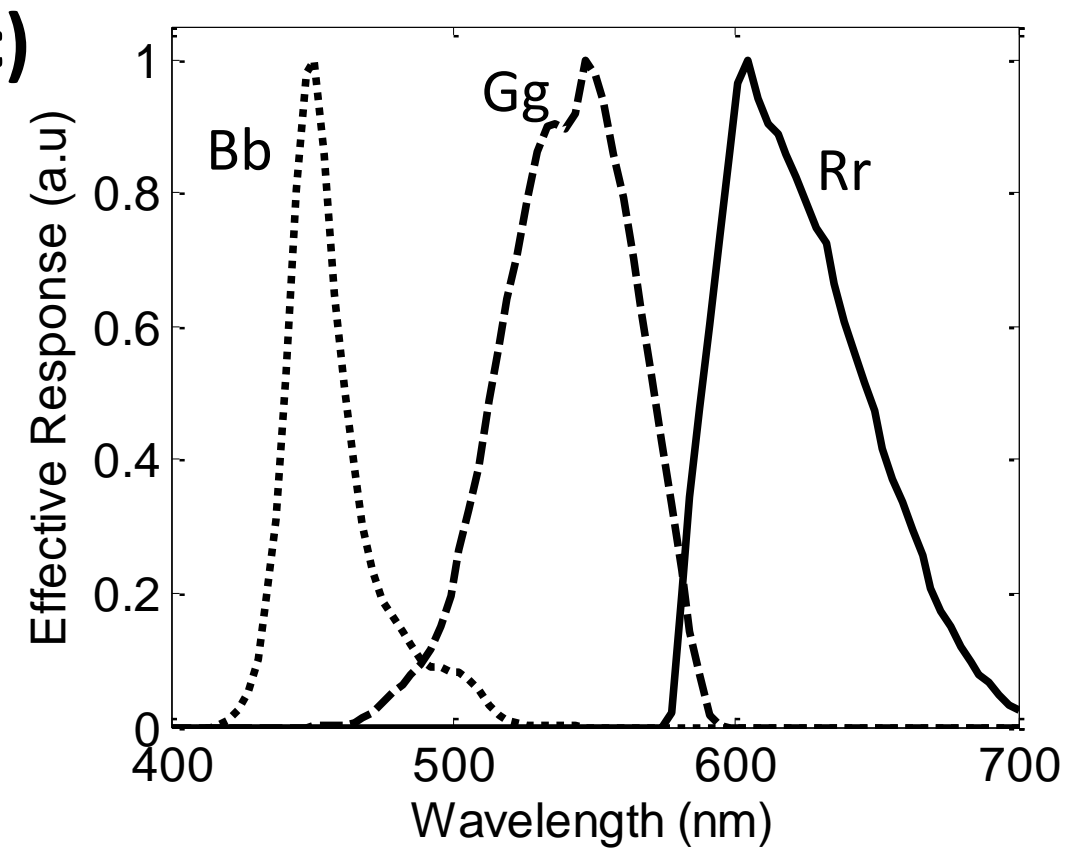

b)

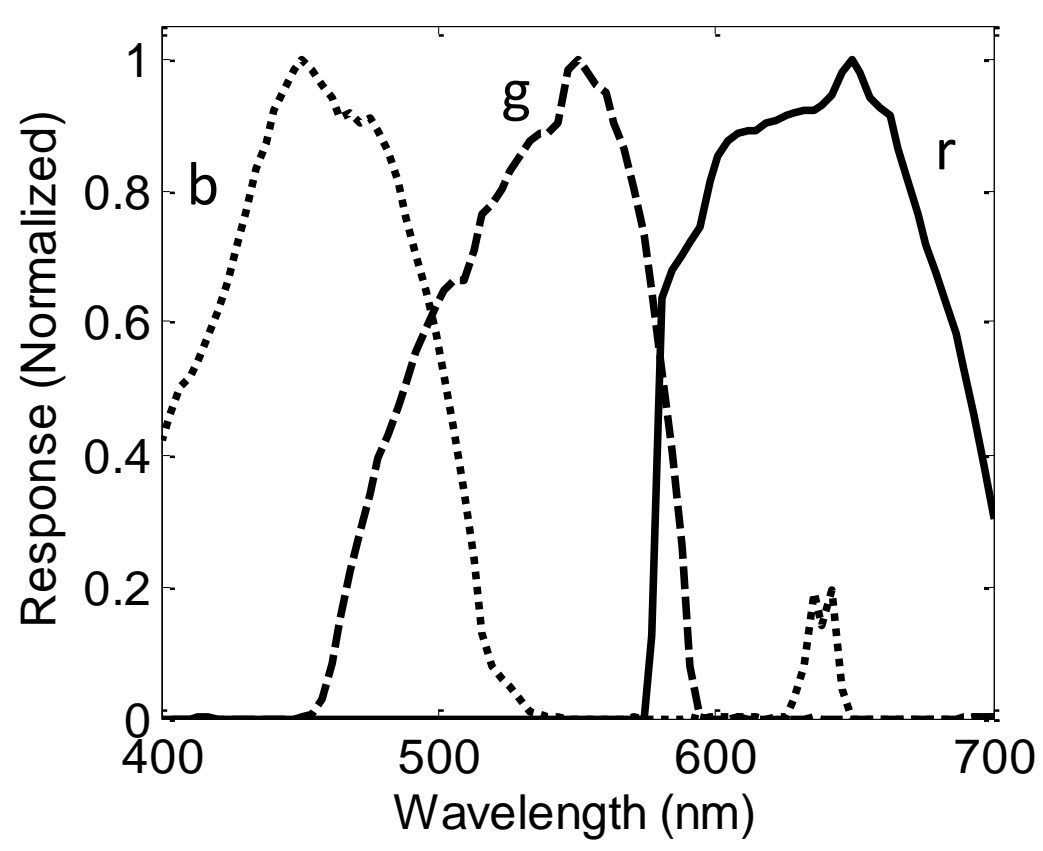

d)

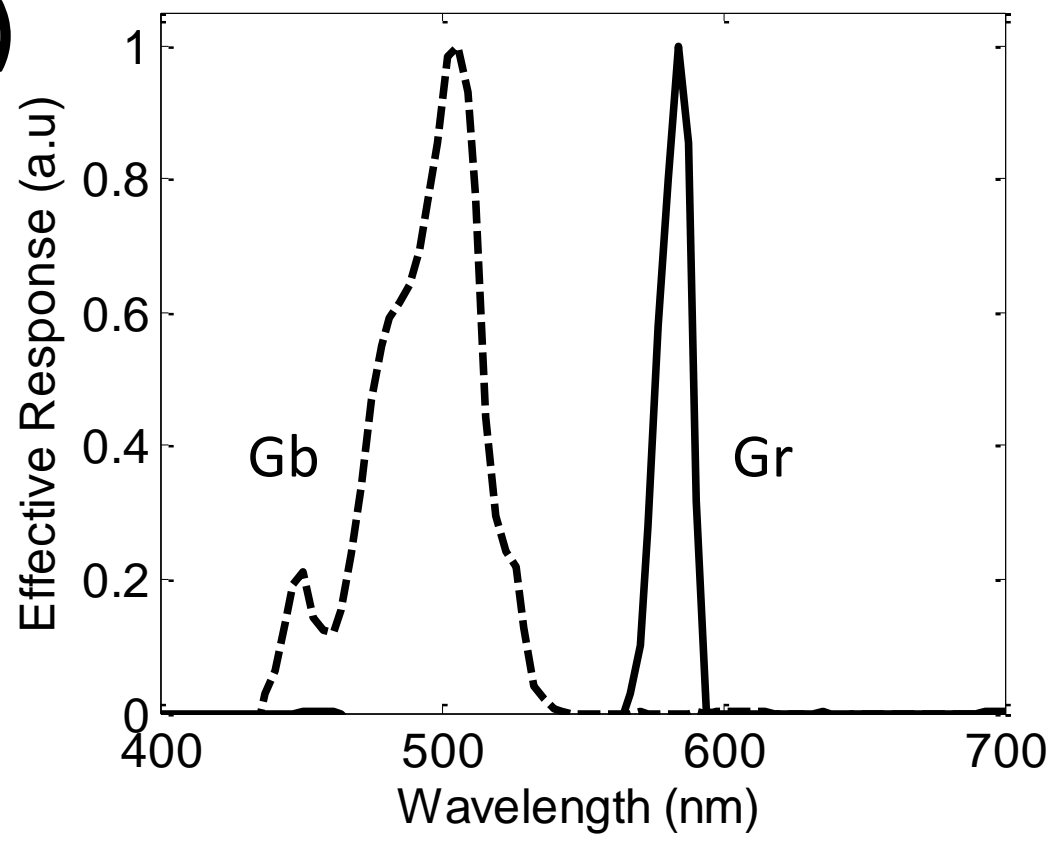




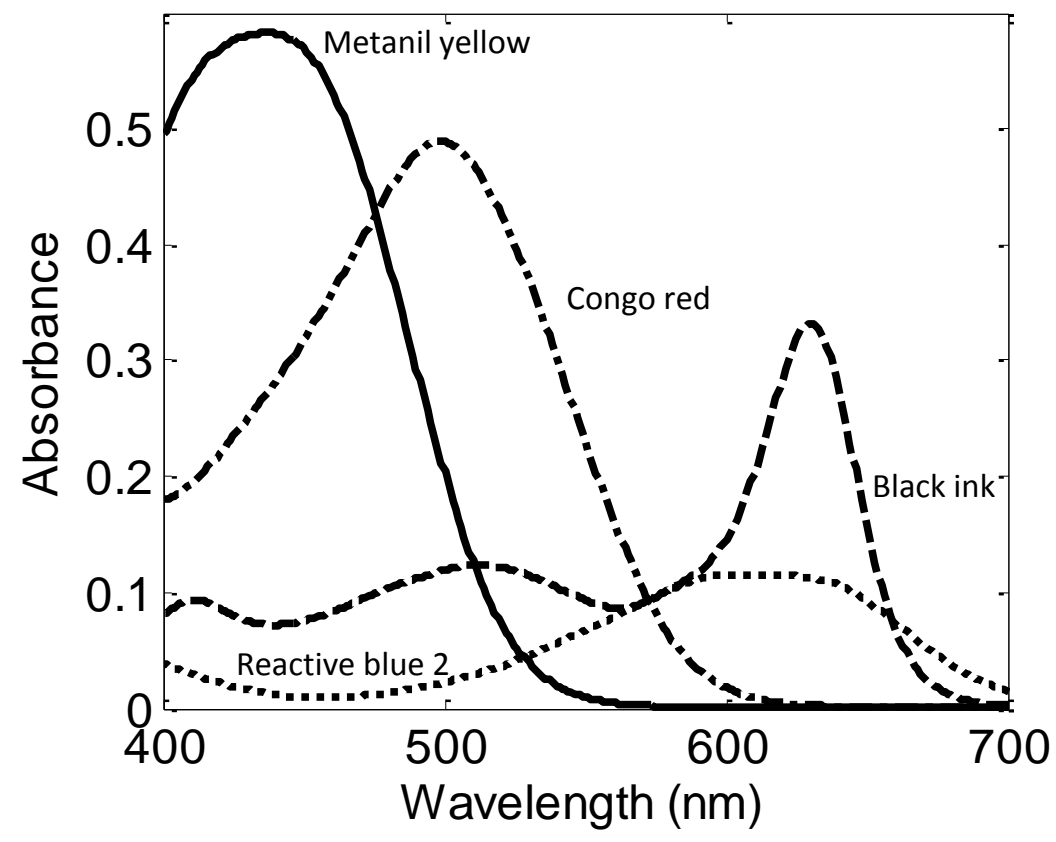



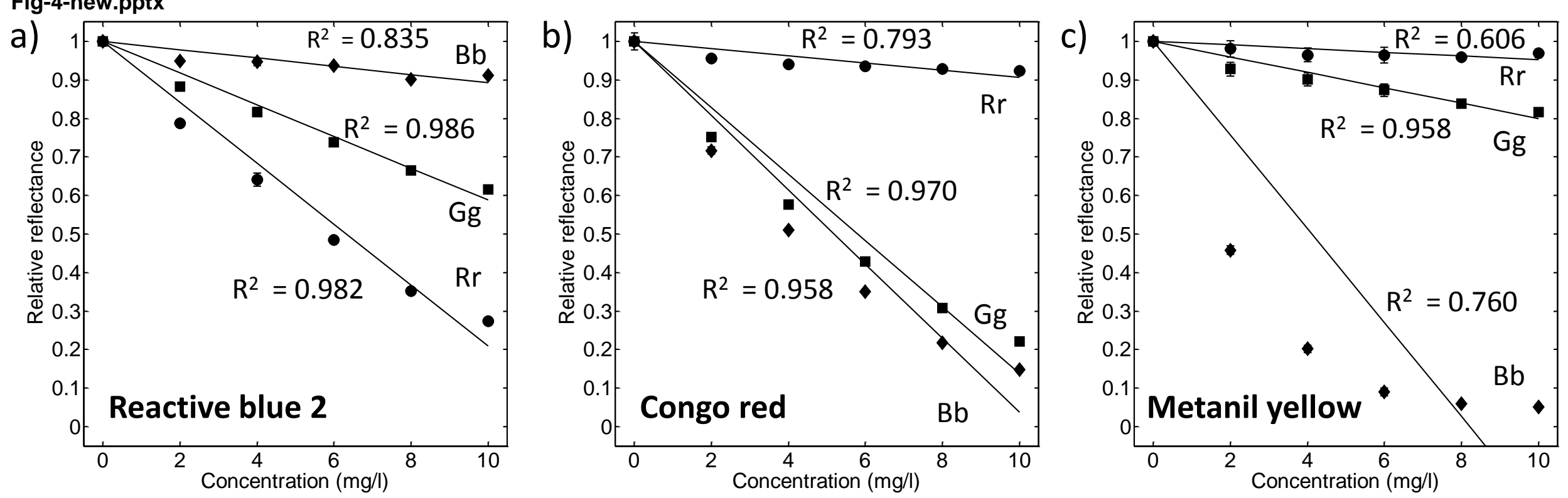

d)

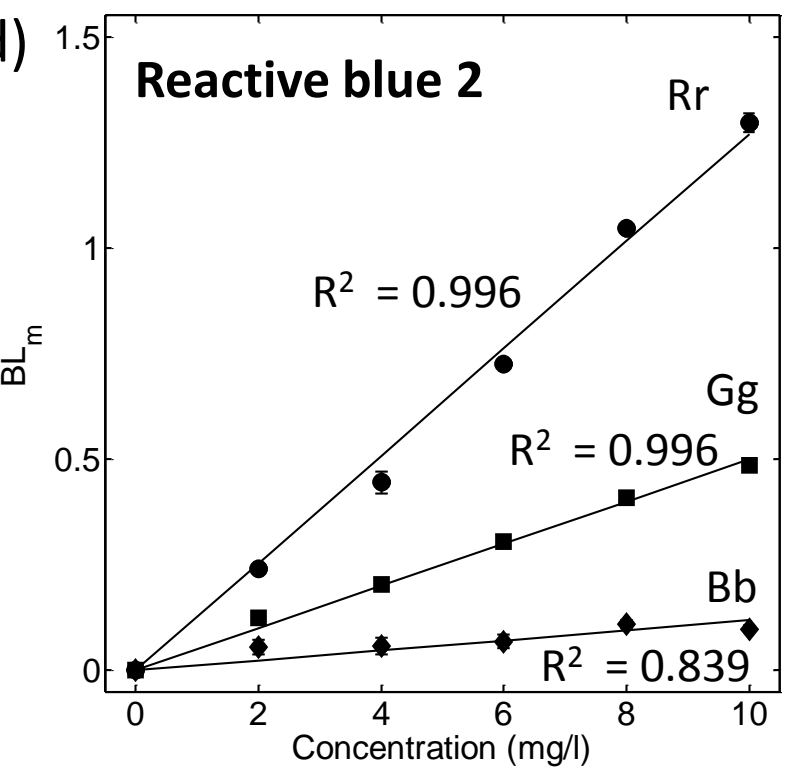

e

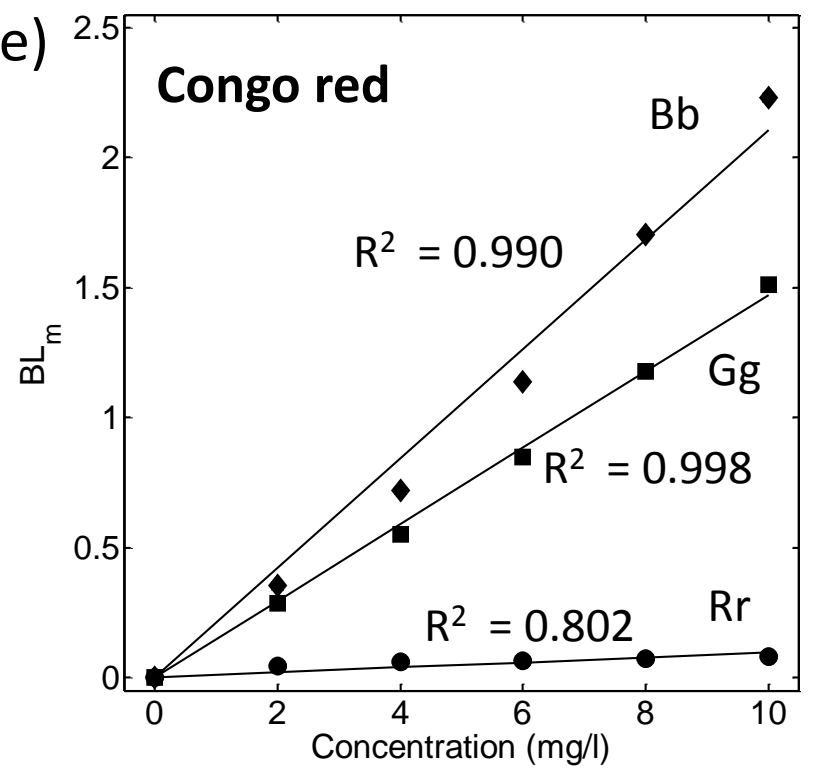

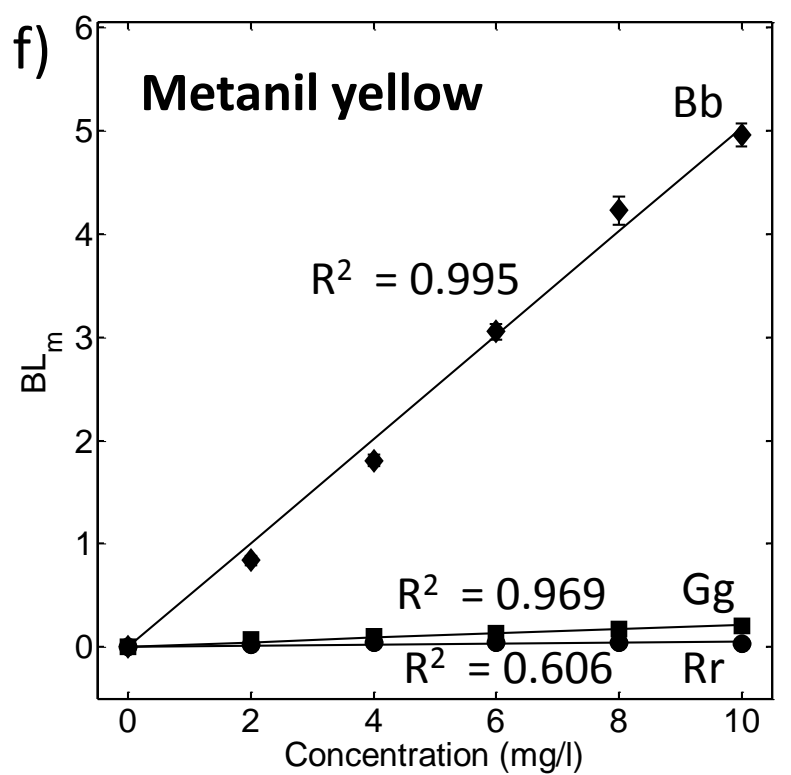




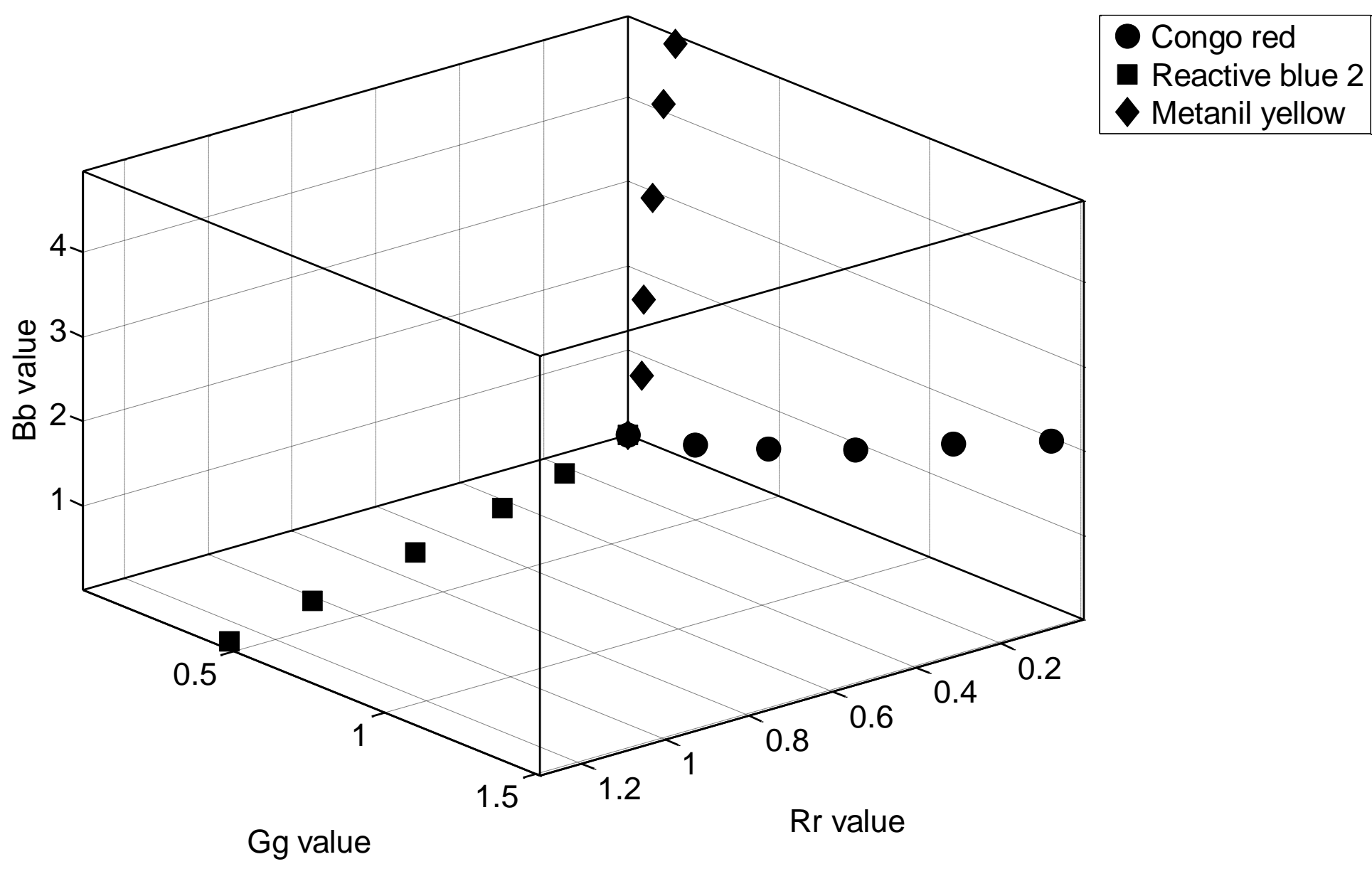




\section{Fig 6 -newepptx}

a)

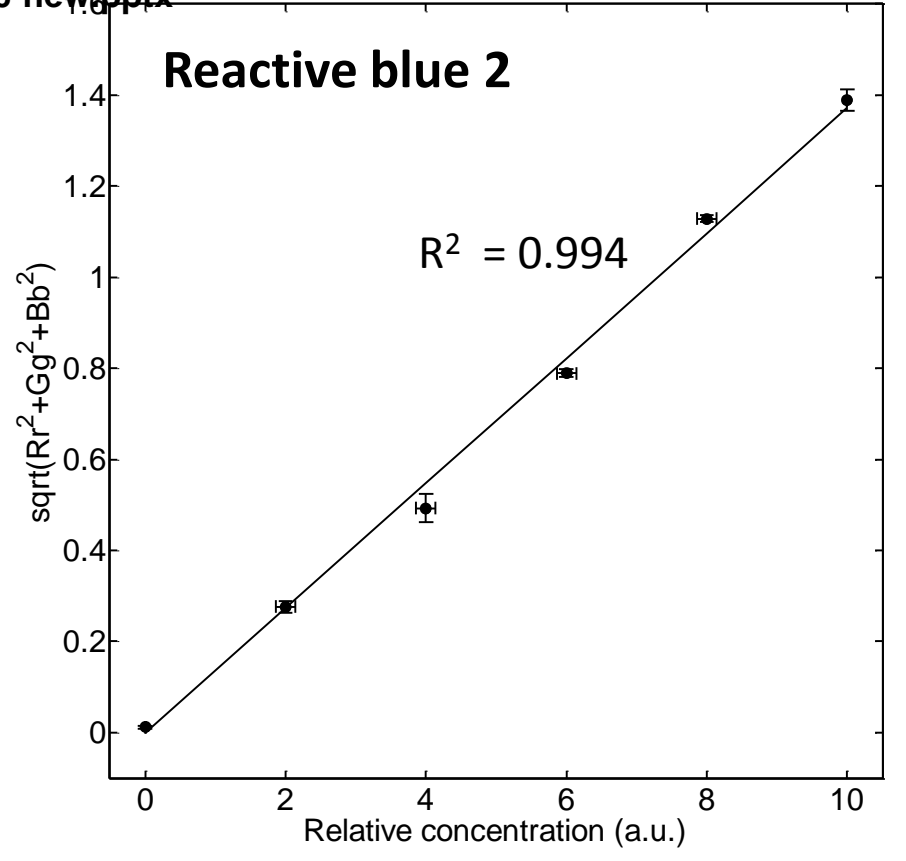

c)

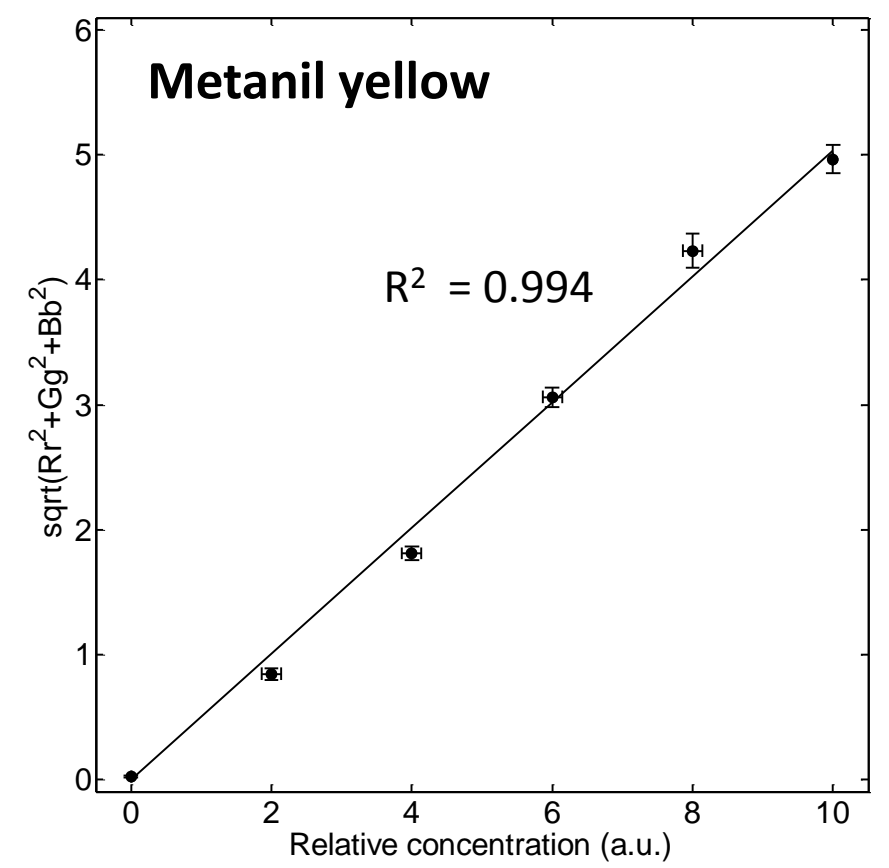

b)

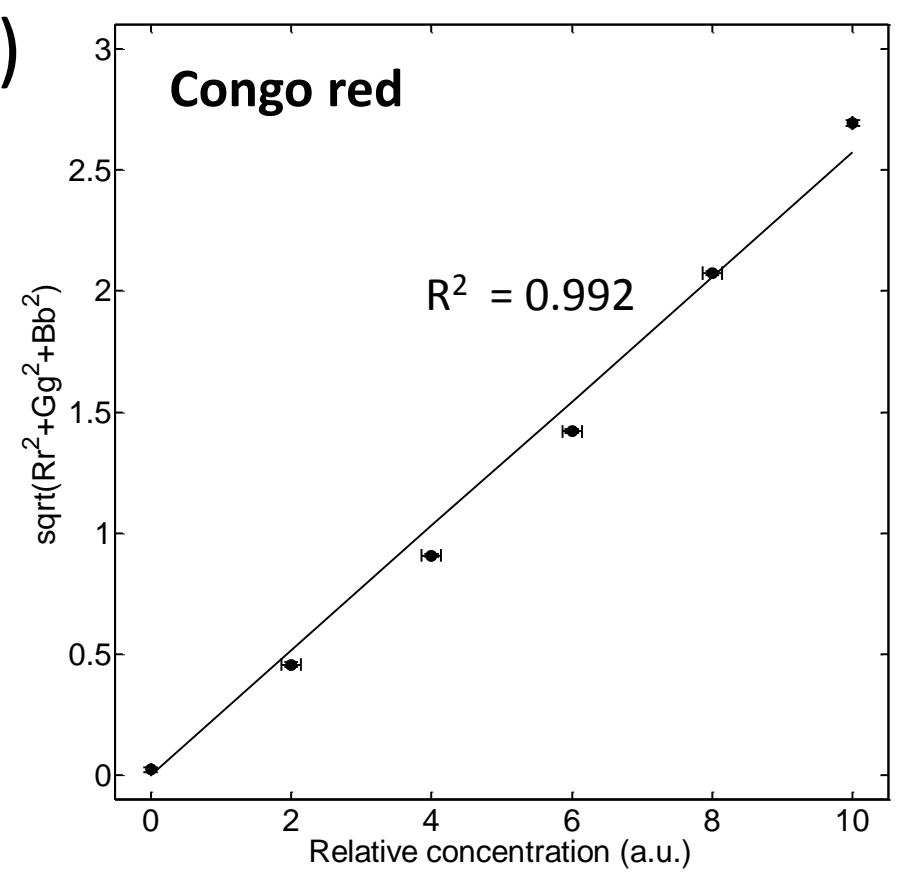

d)

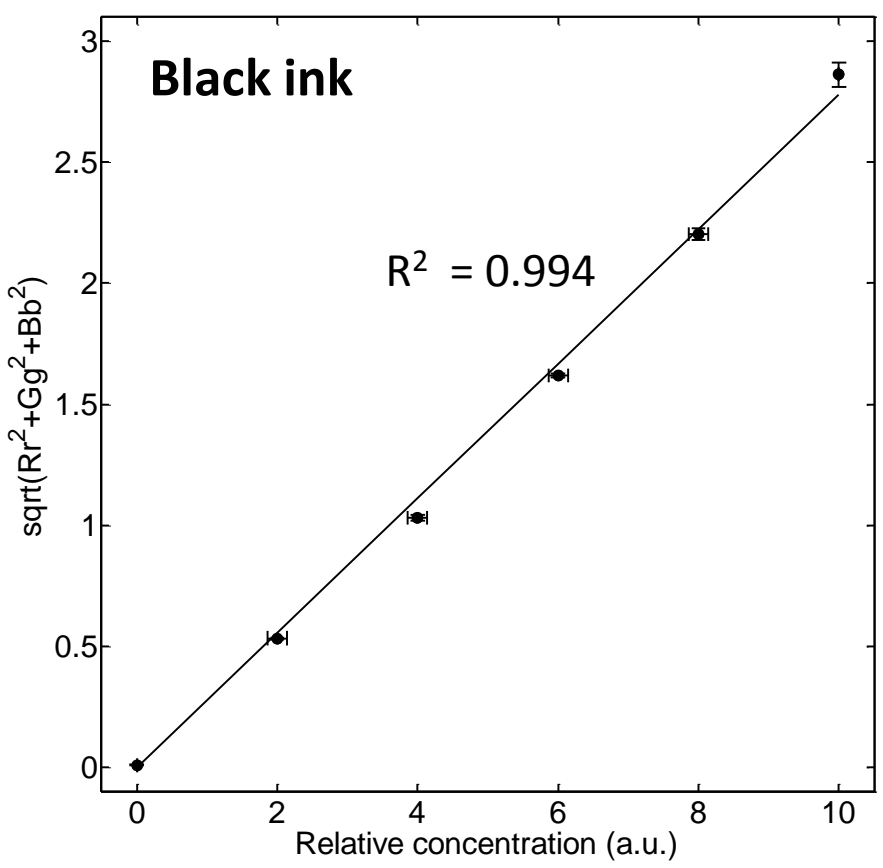



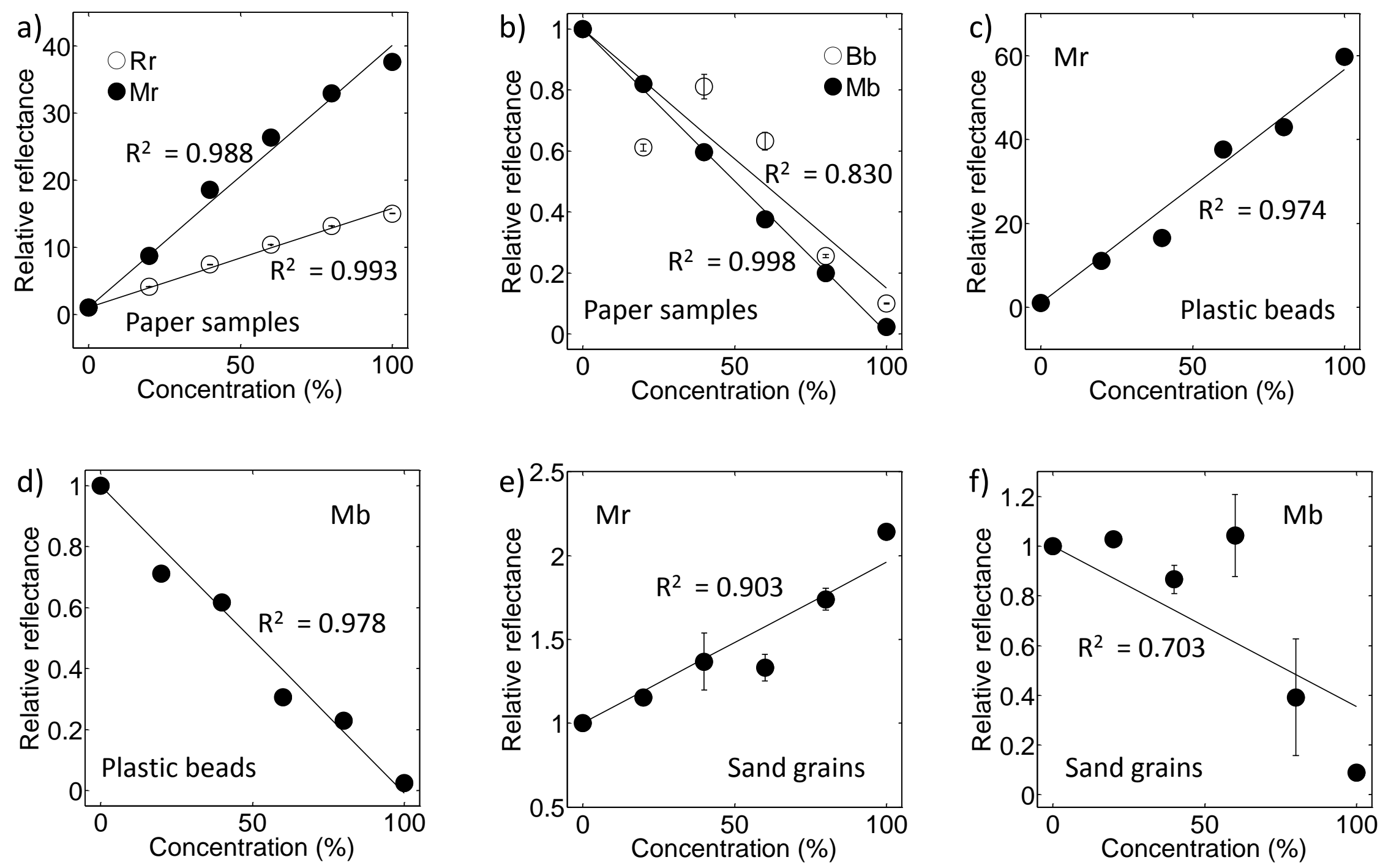


\section{Click here to download Supplementary Material: Iqbal-Eriksson-Highlights.docx}

Supplementary Material
Click here to download S 